\title{
PAPEL ACTUAL DE LA RESONANCIA MAGNÉtICA EN EL ESTADIAJE LOCAL DEL CÁNCER DE PRÓSTATA.
}

Joan Carles Vilanova Busquets, Josep Comet Batlle', Joaquim Barceló Obregón, Miguel Villalón Ortega y Neus Ruscadella Morell.

Departamento de Resonancia Magnética. Clínica Girona. Girona

Departamento de Urologíal. Hospital Universitario Dr. Josep Trueta. Girona. España.

\begin{abstract}
Resumen.- El manejo del cáncer de próstata representa una verdadera problemática clínica, por su historia natural desconocida, impredecible evolución y pronóstico. La variable más importante para el pronóstico de cualquier tumor es el estadiaje. La utilización de los nomogramas, como los de Partin, proporciona información importante en la valoración del estadiaje local. Sin embargo, estos métodos muestran escasa fiabilidad debido a la ausencia de combinar la información anatómica de la glándula. El desarrollo de la técnica de la resonancia magnética (RM), incorporando la valoración anatómica de alta resolución de la próstata conjuntamente con la información metabólica (RM espectroscopía), proporcionan hoy en día el método de imagen de elección para estadiaje del cáncer de próstata, en pacientes seleccionados candidatos a cirugía curativa.
\end{abstract}

La RM de próstata ofrece la valoración más fiable en el estadiaje local y regional. De todas formas, numerosos estudios han descrito una amplia variabilidad en la fiabilidad del estadiaje del cáncer de próstata con $R M$, indicando que la RM no es la técnica de imagen perfecta; al no poder demostrar la afectación microscópica. El objetivo del presente artículo es valorar el papel actual de la RM de próstata en el estadiaje del cáncer de próstata; evaluando las ventajas, limitaciones y las expectativas de futuro utilizando esta tecnología.

Palabras clave: Cáncer de próstata. Estadiaje. Resonancia magnética.

Summary.- The management of prostate cancer represents a real clinical problem for its unknown natural history, and unpredictable outcome and prognosis. The stage is the most important prognostic variable of any tumor. The use of nomograms, such as Partin's tables, supplies important information for the evaluation of the local stage. Nevertheless, these methods have low reliability because they do not use anatomical information of the gland. The development of MRI techniques incorporating high-resolution anatomical evaluation of the prostate in conjunction with metabolic information (MRI spectroscopy) offer the imaging technique of choice for the staging of prostate cancer in selected patients candidates to curative surgery. Prostate MRI offers the most reliable evaluation of local and regional staging. Anyway, many studies have described a wide variability in the reliability of prostate cancer staging by MRI, indicating that MRI is not a perfect imaging test because it cannot detect microscopic involvement. The objective 
of this article is to evaluate the current role of prostatic MRI for prostate cancer staging, evaluating its advantages, limitations and future expectations.

Keywords: Prostate cancer. Staging. MRI.

\section{INTRODUCCIÓN}

En España, el cáncer de próstata es la tercera causa de muerte en hombres por detrás de los tumores de pulmón y colorectales (1). Sin embargo, la tasa de incidencia es considerablemente superior a la mortalidad debido especialmente a que muchos tumores de próstata permanecen latentes. A pesar ello persiste la controversia en los métodos de detección, estadiaje y manejo del cáncer de próstata. No existe un test o técnica de imagen suficientemente fiable para diagnosticar y estadiar el cáncer de próstata. El método de diagnóstico más ampliamente utilizado es la combinación del tacto rectal (TR), niveles del antígeno prostático específico (PSA), biopsia sistemática guiada por ecografía transrectal y valoración del grado Gleason. Los nomogramas desarrollados por Partin incorporan estas variables, conjuntamente con la edad del paciente, proporcionando información importante sobre la estimación del estadiaje local, aunque no incorporan información anatómica de la glándula. Utilizando estos métodos, aproximadamente el $47 \%$ de los hombres sometidos a prostatectomía radical en una población de 3800 pacientes presentaron extensión tumoral extraprostática (2).

El progresivo desarrollo de la técnica de resonancia magnética (RM) se ha producido precisamente debido a las limitaciones de las otras técnicas, con el objetivo de mejorar la fiabilidad en el estadiaje previo al tratamiento, y en particular para demostrar la presencia o ausencia de la extensión extracapsular (3).

Las expectativas iniciales de la ecografía transrectal (ET) como método útil en valorar la extensión extracapsular fueron atenuadas cuando estudios multicéntricos mostraron la escasa fiabilidad de la técnica (4). De igual forma, estudios realizados a finales de los años 1980 con RM mostraron la imposibilidad de diferenciar el cáncer de próstata de la hiperplasia benigna de próstata, y la eficacia en el estadiaje era similar al tacto rectal (5). Sin embargo la introducción de nuevas bobinas (antenas endorectales), mejoras en los equipos de resonancia y mayor experiencia del radiólogo han mostrado un significa- tivo aumento de la eficacia de la $\operatorname{RM}(3,6)$. A pesar del progreso, la extensión microscópica no es posible identificarla por RM, por lo que su uso sigue siendo motivo de discrepancia. Hoy en día no está indicada la RM como método de rutina para estadiaje del cáncer de próstata, pero ofrece una serie de ventajas en pacientes seleccionados (7). Además, el progresivo desarrollo de la tecnología de RM incluyendo estudios combinados empleando contraste endovenoso en secuencias dinámicas, RM espectroscopía y contraste específico para los ganglios linfáticos hacen considerar una mejora en la fiabilidad del estadiaje del cáncer de próstata. En este artículo haremos una revisión actual del papel de la RM en el estadiaje local del cáncer de próstata valorando las ventajas, limitaciones y expectativas del futuro.

\section{Técnica de Resonancia Magnética}

La RM ofrece múltiples ventajas respecto a las otras técnicas de imagen como la tomografía computada o la ecografía transrectal, aunque su disponibilidad es más limitada. Las contraindicaciones de la RM incluyen pacientes con marcapasos, clips intracraneales de aneurisma y fragmentos metálicos intraorbitarios. Los pacientes claustrofóbicos requieren sedación, debido a que los equipos de RM abiertos al mostrar campos magnéticos de baja potencia no son adecuados para un estudio fiable de la pelvis. La técnica de RM se ha beneficiado de un importante progreso en la tecnología, mejorando las antenas (bobinas) y las secuencias de adquisición para obtener estudios de alta resolución. El progresivo desarrollo ha permitido utilizar una bobina endorectal en combinación a una bobina pélvica, permitiendo así un estudio completo y eficaz de toda la pelvis.

El protocolo de estudio incluye realizar secuencias potenciadas en $\mathrm{Tl}$ de toda la pelvis, desde la bifurcación aórtica hasta la sínfisis púbica para valorar afectación ósea y la presencia de adenopatías. Posteriormente se realiza estudio de alta resolución de la región prostática en los tres planos del espacio en secuencias T2. Las secuencias en supresión grasa no se han mostrado de eficacia superior en la evaluación de la próstata (8).

La RM ofrece la posibilidad de evaluar no solamente la anatomía prostática, sino que además es posible obtener información metabólica de la glándula mediante curvas de espectroscopía con una secuencia específica (9).

Además, de forma opcional es posible administrar contraste endovenoso, gadolinio, de forma dinámica para incluso mejorar la capacidad de de- 
tección del tumor; aunque ello no se utilice de forma generalizada (10).

\section{Imagen en RM del Cáncer de Próstata}

Las secuencias potenciadas en $\mathrm{Tl}$ muestran una señal intermedia de la glándula prostática y vesículas seminales que puede diferenciarse de la hiperseñal de la grasa periprostática (Figura 1). Al mismo tiempo la secuencia en $\mathrm{Tl}$ permite valorar la existencia de hemorragia postbiopsia glandular, adenopatías pélvicas (Figura 2) y metástasis óseas (Figura 3). Para evitar imágenes en RM que puedan simular carcinoma es imprescindible realizar el estudio de $\mathrm{RM}$ en un tiempo no inferior a 3 semanas $y$ en caso

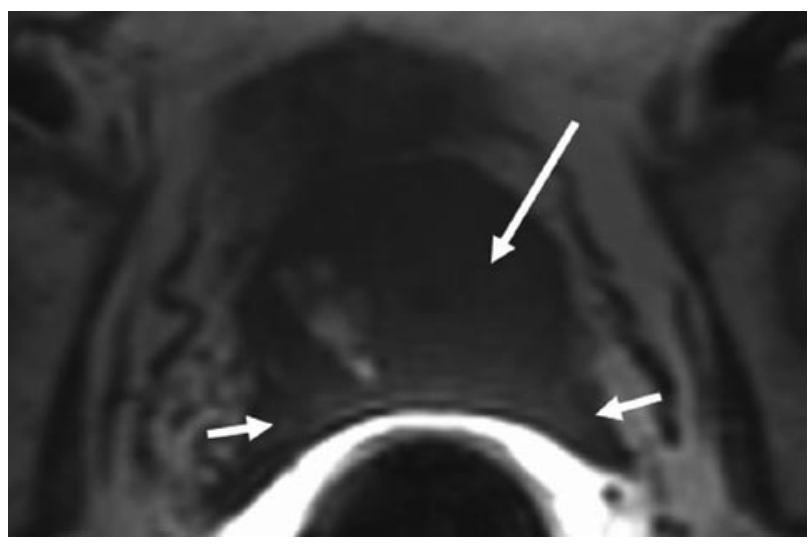

FIGURA 1. RM corte axial en T1. La glándula muestra señal isointensa (flecha larga) sin poder diferenciar

lesiones nodulares. Las vesículas seminales muestran la misma señal isointensa (flechas cortas)

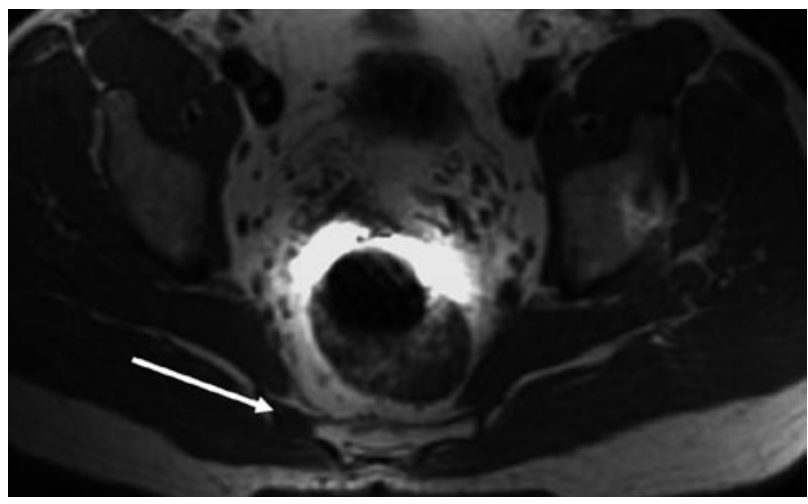

FIGURA 3. RM corte axial de la pelvis. Lesión metastásica del sacro (flecha) detectada en la valoración prequirúrgica de paciente con neoplasia de próstata (PSA: 7,3 ng/ml, Gleason 5). de realizarse un estudio de espectroscopia es preciso esperar un intervalo de tiempo entre 6-8 semanas postbiopsia.

Las secuencias potenciadas en T2 permiten realizar una valoración de la anatomía zonal de la próstata (Figura 4). La señal RM de la glándula periférica en secuencias potenciadas en T2 es hiperintensa y homogénea. La glándula central es hipointensa y heterogénea; comprimiendo la glándula periférica en caso de hipertrofia. Las vesículas seminales se muestran con hiper señal en RM de aspecto multilocular quístico con paredes finas hipointensas (Figura 5).

La mayoría de los tumores de próstata se originan en la glándula periférica, mostrándose como

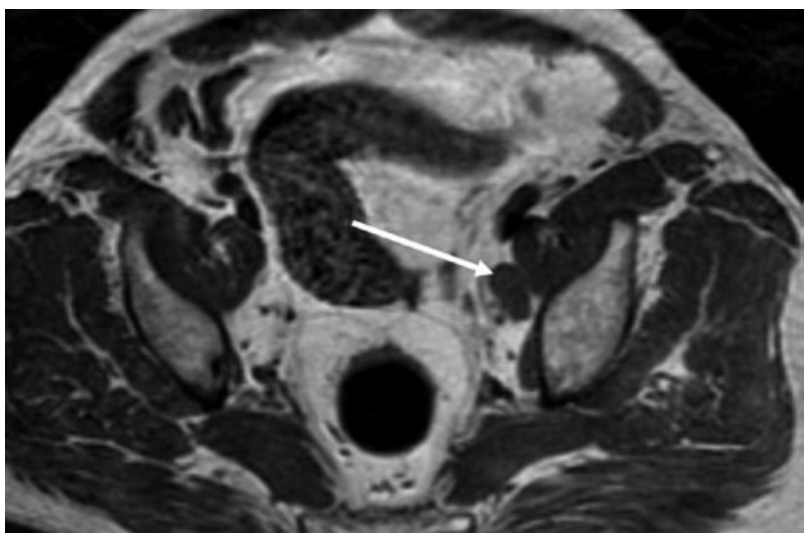

FIGURA 2. RM corte axial de la pelvis. Adenopatía metastásica de la cadena ilíaca izquierda (flecha).

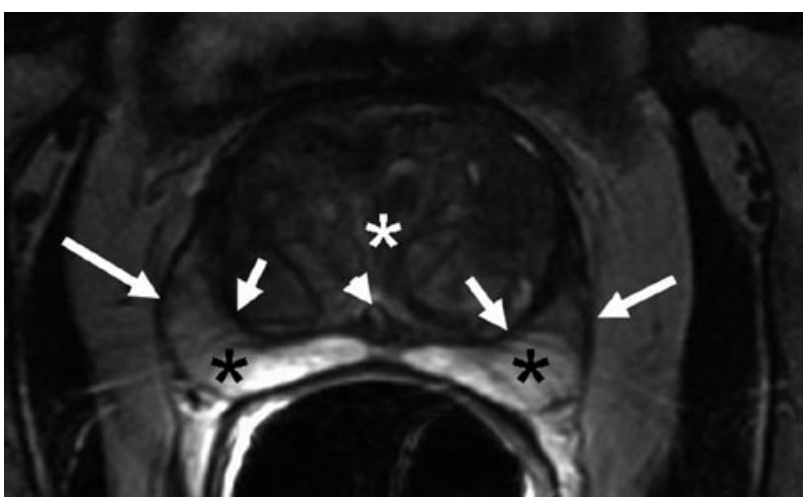

FIGURA 4. RM corte axial en T2. La glándula periférica es hiperintensa (asteriscos negros) separada de la glándula central por la cápsula quirúrgica (flechas cortas). La cápsula glandular se muestra como una fina banda hipointensa (flechas largas). La glándula central es heterogénea e hipointensa en relación a hipertrofia benigna (asterisco blanco). Uretra (cabeza de flecha). 


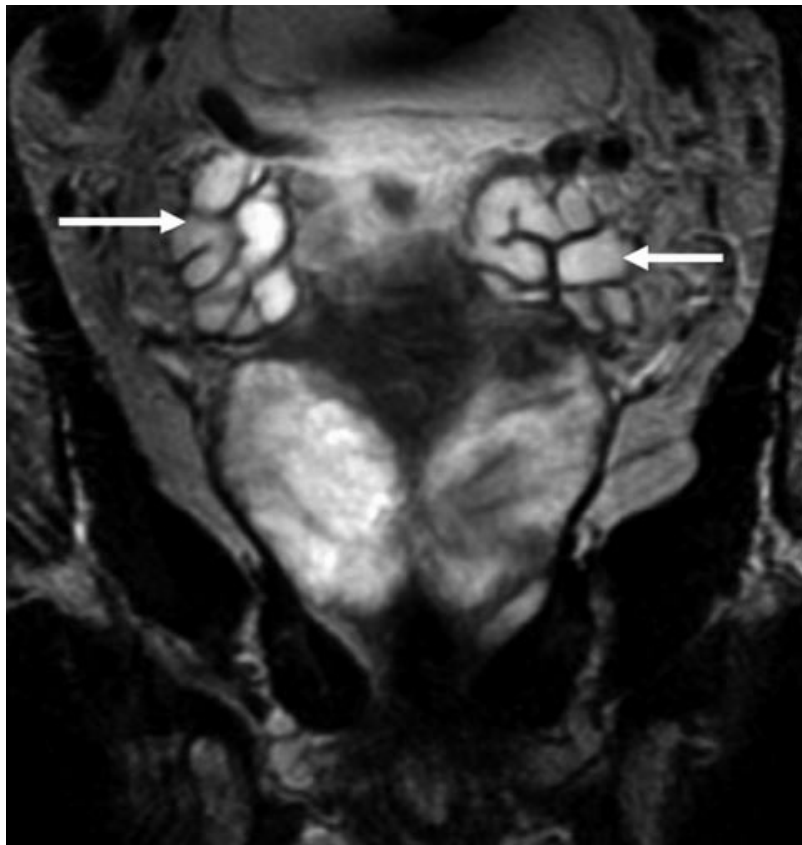

FIGURA 5. RM corte coronal en T2. Las vesículas seminales (flechas) muestran una señal hiperintensa en aspecto multiloculado, en racimos con septos hipointensos.

hiposeñal focal en secuencias potenciadas en T2 (Figura 6). De todas formas la hiposeñal en RM es poco específico debido a que otras lesiones como la prostatitis, cicatrices, hiperplasia (Figura 7), hemorragia postbiopsia o calcificaciones pueden mostrar un aspecto similar (11). El aspecto del cáncer de próstata en la glándula central, generalmente, no puede diferenciarse del tejido normal al mostrar en secuencias T2 un aspecto similar la hiperplasia y el cáncer (Figura 8) (12).

El estudio de espectroscopía en RM permite mejorar la especificidad al poder combinar la información anatómica y metabólica en único estudio (9). En la próstata se obtienen básicamente tres metabolitos, creatina, colina y citrato. El tumor prostático muestra una elevación significativa de la colina y niveles bajos del citrato respecto a la elevación del citrato y bajo nivel de colina en el tejido normal (Figura 6). Los niveles de creatina se utilizan al mantenerse constante tanto en el tejido normal como neoplásico. De aquí que se utilice el cociente: creatina $(\mathrm{Cr})+$ colina $(C)$ respecto al valor del citrato $(\mathrm{Ci})(\mathrm{Cr}+\mathrm{C} / \mathrm{Ci})$. Cocientes elevados indicarían neoplasia y niveles bajos indicarían benignidad (9).

\section{Estadiaje}

El objetivo del estadiaje local es la selección del abordaje terapéutico del cáncer en caso de que el tumor sea intracapsular (estadio T2) y posible curación, o si el tumor es extracapsular (estadio T3) aplicar tratamiento paliativo.

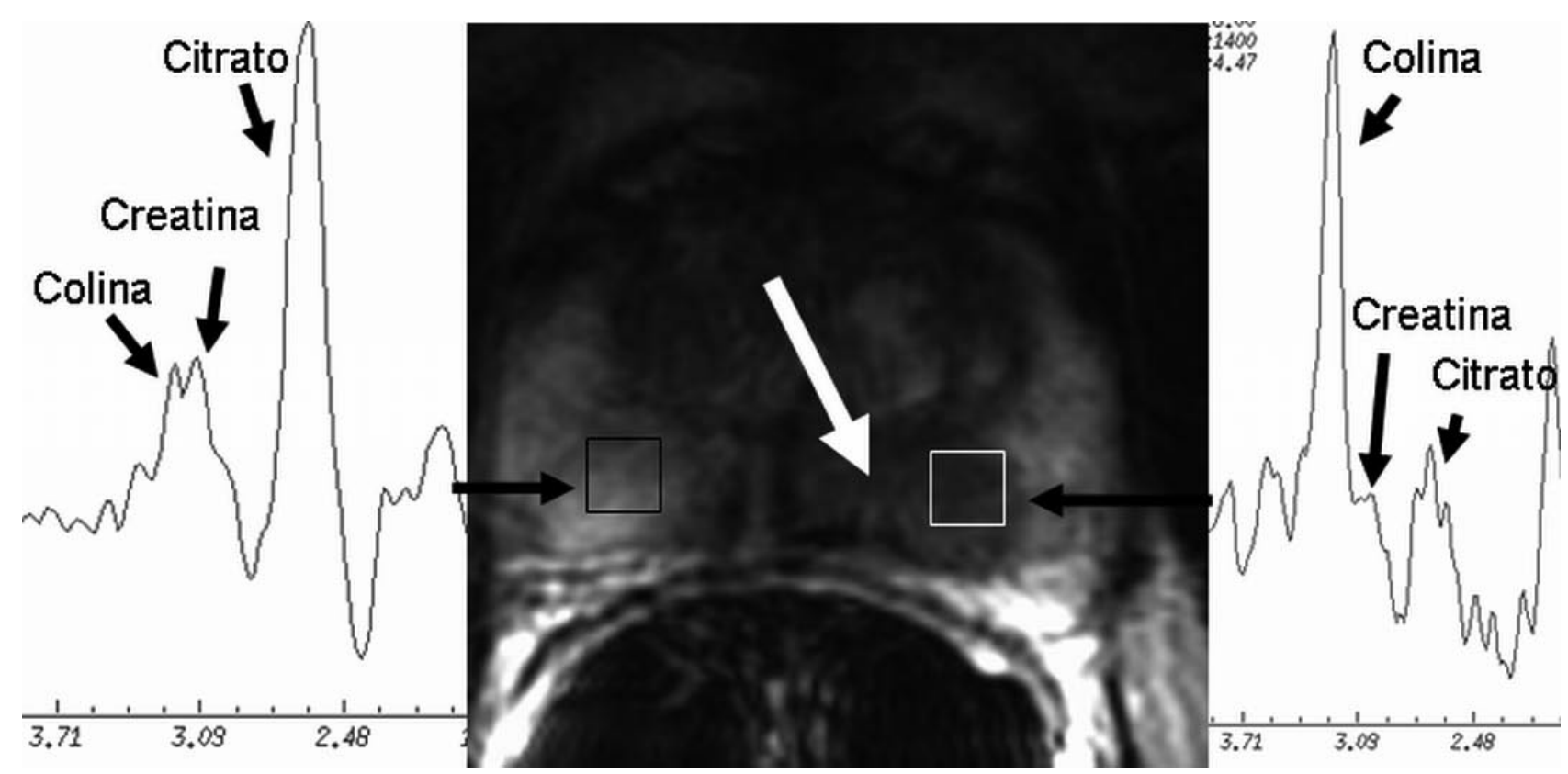

FIGURA 6. RM corte axial en T2 y curvas de espectroscopía. La glándula periférica normal muestra señal hiperintensa con elevación del citrato y reducción de la colina (cuadrado negro). El cáncer muestra hiposeñal en la glándula periférica (flecha blanca) y perfil metabólico con elevación de la colina y reducción del citrato. 
La técnica de imagen de elección para estadiaje del cáncer de próstata es la RM por su capacidad de identificar mejor la morfología anatómica de la glándula. Las técnicas como el tacto rectal, ecografía transrectal y tomografía computarizada son poco fiables para un correcto estadiaje local del cáncer de próstata (13). La RM se muestra como la técnica más fiable para el estadiaje del cáncer de próstata, comparativamente con la tomografía computarizada, ecografía y el tacto rectal (14).

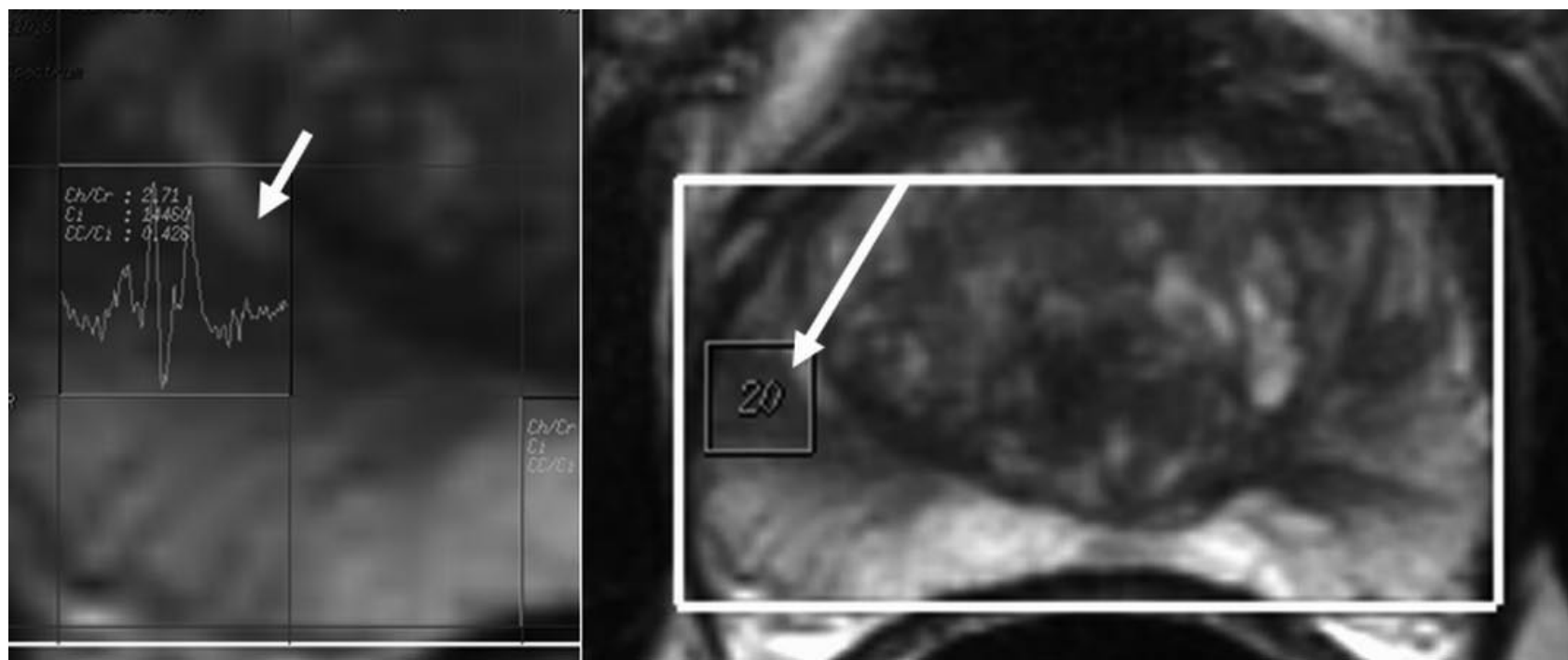

FIGURA 7. RM corte axial en T2. Lesión hipointensa en la glándula periférica derecha del paciente (flecha larga) sugestiva de cáncer, con curva normal de espectroscopía (flecha corta). La biopsia demostró hiperplasia.
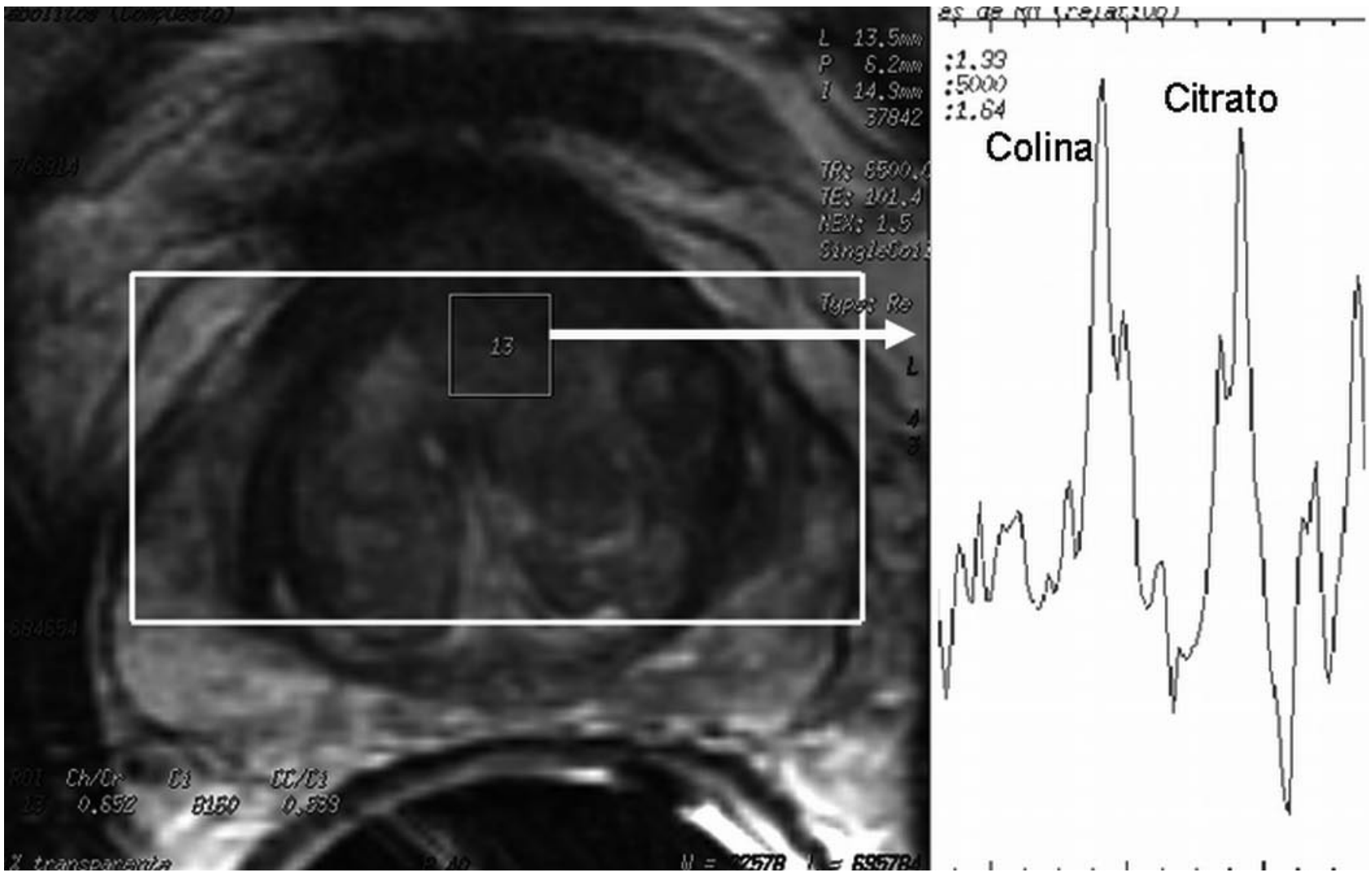

FIGURA 8. RM corte axial en T2. Imagen en RM sin alteraciones en la señal con glándula central heterogénea de forma normal. La curva de espectroscopía muestra elevación de la colina indicativo de neoplasia. La biopsia demostró carcinoma 


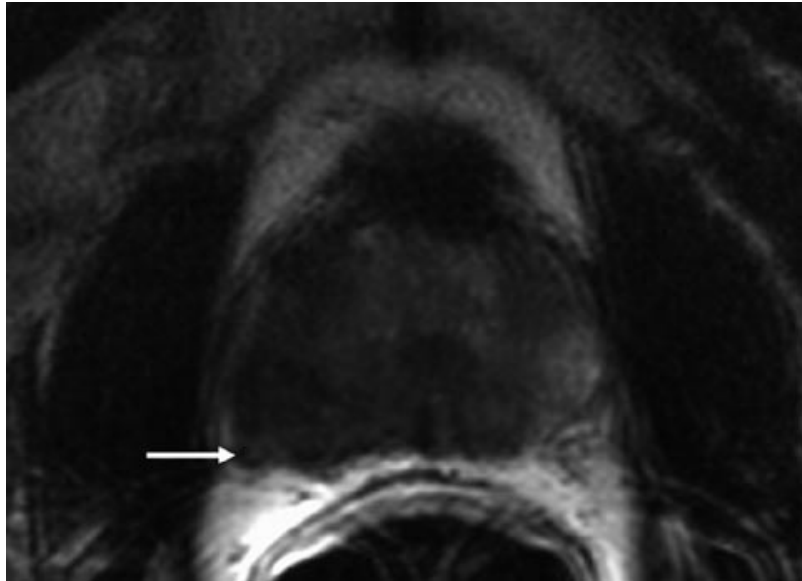

FIGURA 9. RM corte axial en T2. Neoplasia de la glándula periférica derecha en extensión contralateral con obliteración de la línea de la cápsula y espiculación (flecha) sobre la grasa periprostática.

Los criterios utilizados para considerar extensión extracapsular del tumor prostático en RM incluyen (15): protrusión focal irregular-espiculada de la cápsula (Figura 9), pérdida de la hiposeñal normal de la cápsula, obliteración del ángulo rectoprostático (Figura 10), asimetría y afectación del plexo neurovacular (Figura 11), y extensión del tumor a vesículas seminales (Tabla). La invasión de la vesícula seminal se demuestra por la presencia de hiposeñal en el seno de las vesículas (Figura 12).

Para obtener la máxima eficacia en el estadiaje del cáncer de próstata en RM es imprescindible utilizar bobina endorectal o en su defecto bobina de

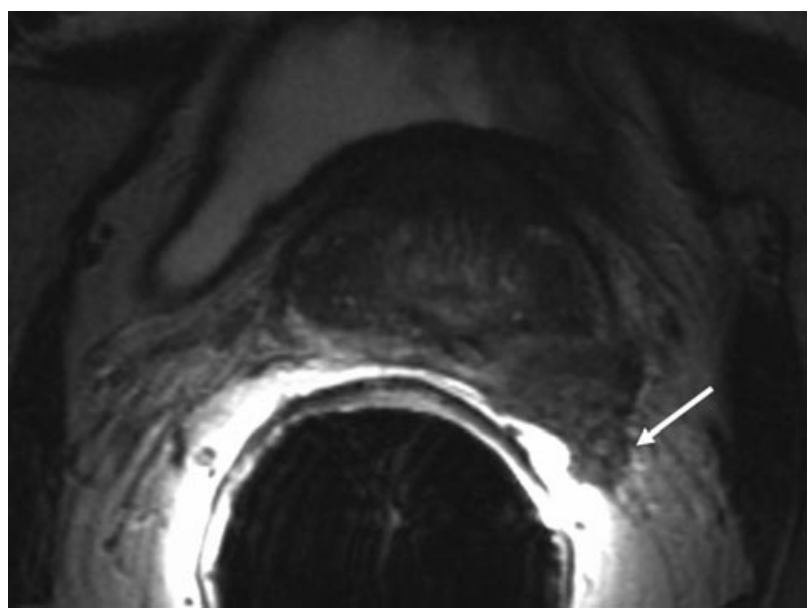

FIGURA 1 1. RM corte axial en T2. Neoplasia de la glándula periférica izquierda con infiltración del plexo neurovascular (flecha).

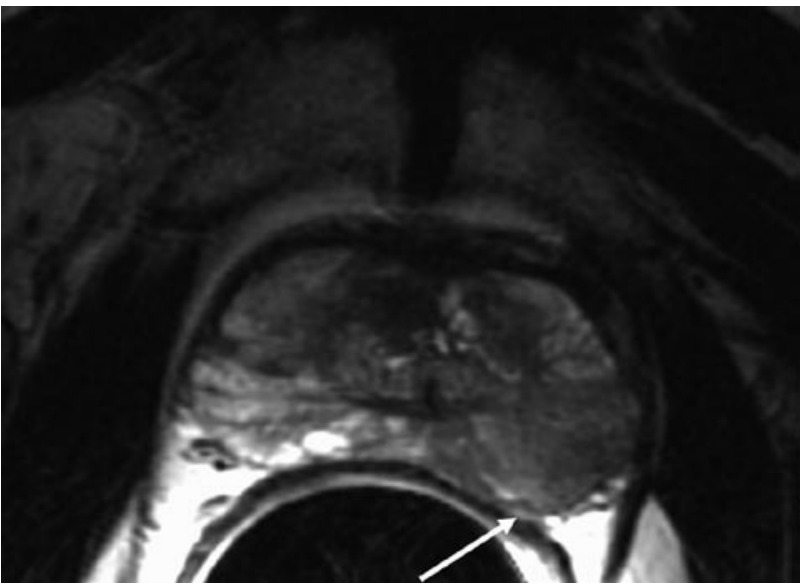

FIGURA 10. RM corte axial en T2. Cáncer de próstata de la glándula periférica izquierda con protrusión capsular y rectificación del ángulo rectoprostático (flecha).

pelvis multicanal para poder obtener estudios de alta resolución de la pelvis (16).

Es importante conocer que después de la biopsia puede observarse irregularidad de la cápsula (Figura 13), sin que ello sea indicativo de extensión capsular (17).

Es difícil valorar de forma objetiva los resultados publicados sobre la fiabilidad de la RM en el estadiaje local del cáncer de próstata, debido a las distintas técnicas y métodos utilizados. De todas formas, según un meta-análisis realizado sobre 74 estudios publicados en relación al estadiaje del cáncer de próstata se concluye que los mejores resultados se obtienen realizando el examen RM de la próstata con secuencias de alta resolución en múltiples planos utilizando bobina endorectal (18). El criterio más específico de extensión extracapsular (EEC) es la asimetría del plexo neurovascular (sensibilidad 38\%,

\section{TABLA. CRITERIOS EN RM DE EXTENSIÓN EXTRACAPSULAR}

- Protrusión focal irregular, espiculada de la cápsula

- Pérdida de la hiposeñal normal de la cápsula

- Obliteración del ángulo rectoprostático

- Afectación o asimetría del plexo neurovacular, grasa periprostática

- Extensión a vesículas seminales 


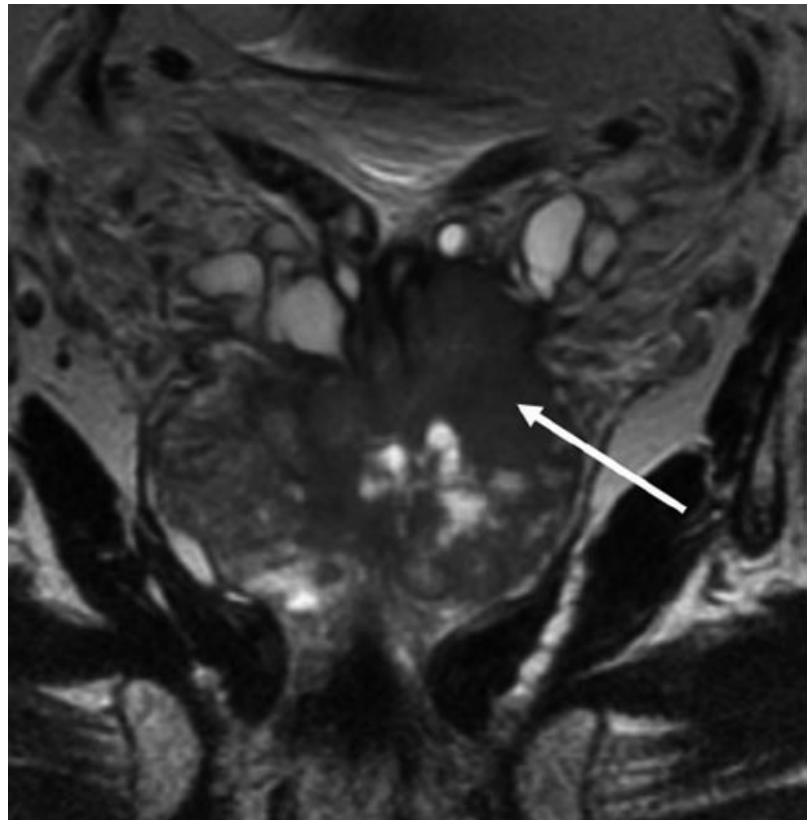

FIGURA 12. RM corte coronal en T2. Neoplasia de la base prostática izquierda infiltrando la vesícula seminal (flecha).

especificidad $95 \%$ ) y el más sensible es la valoración global de todos los criterios de EEC (sensibilidad $68 \%$, especificidad $72 \%$ ) (18).

Los estudios que han analizado los criterios de selección para realizar un estudio en RM para estadiaje del cáncer de próstata, sugieren incluir pacientes con riesgo intermedio de tener estadio $\mathrm{T} 3$ (PSA, 10-20ng/ml, Gleason 5-7) (19). Esta aproximación se ha mostrado también de coste/eficacia tanto para los pacientes de riesgo intermedio como para los de riesgo elevado de tener extensión extracapsular (20).

Se ha demostrado el valor añadido de la RM endorectal en la predicción del cáncer de próstata intraglandular para todos los grupos de riesgo (21) y especialmente en los grupos de riesgo elevado comparativamente con las tablas de Partin (22).

A pesar del excelente contraste de los tejidos de partes blandas por parte de la RM, la técnica tiene valor limitado en la valoración metastásica ganglionar; siendo la fiabilidad similar a la tomografía computada. De todas formas un estudio reciente demuestra que la combinación de las tablas de Partin con la información en conjunto de la RM para extensión extracapsular, vesículas seminales y metástasis ganglionar ofrece un elevado valor predictivo para el diagnóstico de metástasis ganglionar (23).

\section{Nuevas técnicas de resonancia magnética}

\section{RM espectroscopía}

La RM espectroscopía utiliza la tecnología de forma similar a la RM convencional pero, más que información anatómica, proporciona información funcional en relación a los componentes metabólicos de la glándula. Se ha descrito la técnica brevemente en el apartado de técnica RM y de forma más exhaustiva en otro capítulo de esta monografía.

La aplicación de la espectroscopía en RM mejora la fiabilidad del examen en el estadiaje preoperatorio (especificidad del $90 \%$ cuando ambas técnicas indican cáncer), en la localización del cáncer y en el seguimiento post-tratamiento $(14 ; 24)$. Además, la RM espectroscopía también reduce la variabilidad inter-observador en el análisis de la de la extensión del cáncer de próstata por RM (24).

La RM con espectroscopía proporciona un valor adicional a los nomogramas clínicos de Partin en la predicción de la extensión extracapsular de cáncer. Estudios recientes muestran un incremento en la fiabilidad del estadiaje del cáncer de próstata utilizando la combinación de los nomogramas clínicos conjuntamente a la información de la RM espectroscopía, respecto a la información únicamente de los nomogramas clínicos, siendo del $88 \%$ y $80 \%$ respectivamente, siendo más significativo en el grupo de riesgo elevado (25).

\section{RM dinámica con contraste}

La RM utilizando secuencias rápidas tras la administración de contraste endovenoso (gadolinio)

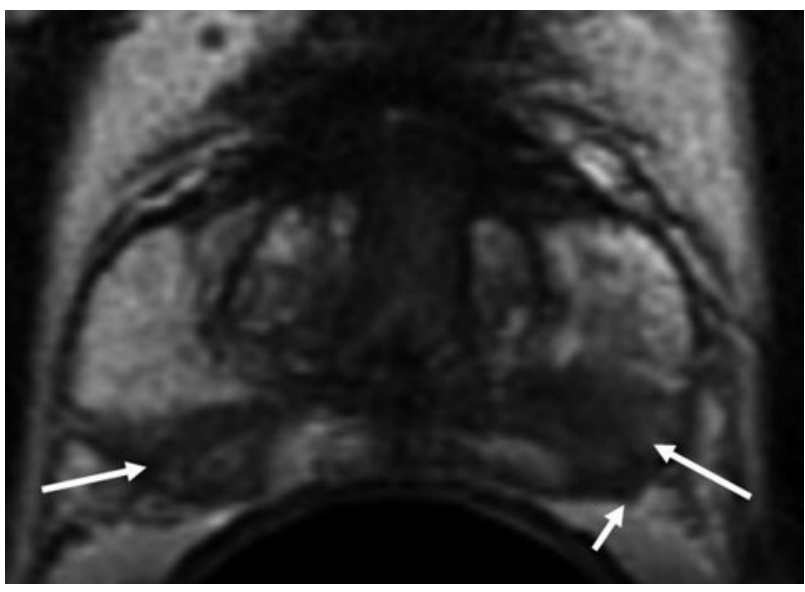

FIGURA 13. RM corte axial en T2. Neoplasia de próstata bilateral (flechas) con espiculación de la glándula izquierda (flecha pequeña) 4 semanas post-biopsia, que podría sugerir estadio T3. La anatomía patológica confirmó estadio T2. 
permite valorar la vascularización de la glándula de forma dinámica. Estudios preliminares han mostrado una intensa captación del contraste en el primer minuto tras su administración en el tejido neoplásico comparativamente con la glándula normal indicativo de angiogénesis del tumor (26). Ogura y cols. (27) demuestran una fiabilidad del $84 \%$ en el estadiaje local del cáncer de próstata utilizando la técnica dinámica con contraste. Estos resultados no difieren de los resultados publicados utilizando la RM sin contraste endovenoso. Es preciso realizar más estudios y mejoras en la tecnología para valorar la posible utilidad de la técnica con contraste endovenoso en el estudio por RM del cáncer de próstata.

\section{RM linfografía}

La extensión ganglionar del tumor modifica el estadiaje y secundariamente la opción terapéutica y pronóstico. Las técnicas de imagen como la tomografía computarizada y la RM utilizan criterios del ta-

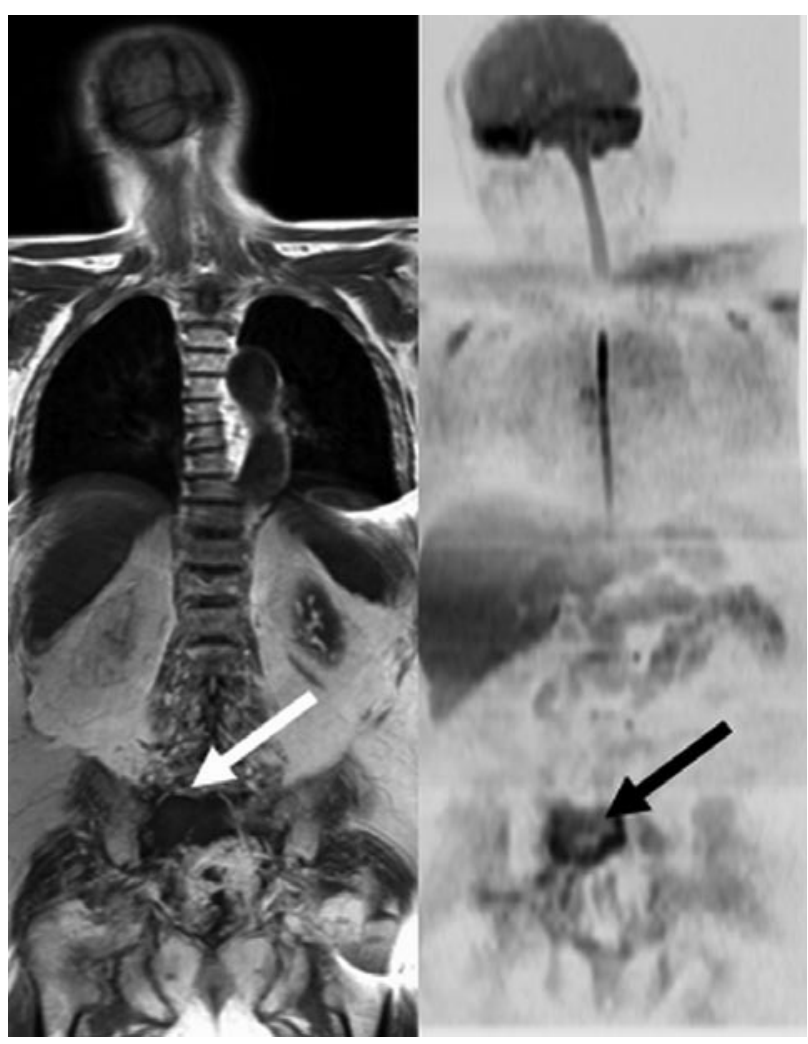

FIGURA 14. RM de cuerpo entero (Whole body). Paciente son sospecha de lesión postraumática en sacro por gammagrafía. La RM de cuerpo entero en Tl demuestra la lesión infiltrativa del sacro derecho (flecha blanca). La nueva secuencia de difusión por RM en imagen de PET-virtual confirma la lesión metastásica (flecha negra). maño ganglionar para indicar la existencia de metástasis. No existe un criterio unificado para indicar el nivel de corte del tamaño ganglionar, pero se acepta como valor máximo el de $10 \mathrm{~mm}$ medido en el eje corto del ganglio (6). Para mejorar la fiabilidad se han desarrollado unos contrastes órgano-específicos (28). Se inyectan micro partículas de óxido de hierro súper-paramagnéticas (USPIO) de forma endovenosa que son fagocitadas por los macrófagos del sistema reticuloendotelial normal del ganglio, donde la señal RM se muestra hipointensa. Los nódulos metastásicos, donde los macrófagos normales son reemplazados por el tumor, no captan las partículas de hierro y en consecuencia no existe alteración en la señal RM. La experiencia inicial sugiere que el contraste USPIO utilizando el método de RM linfografía mejora la sensibilidad y especificidad para la detección ganglionar metastásica (28).

\section{RM de cuerpo entero}

La caracterización del tejido de partes blandas por RM es superior en la sensibilidad y especificidad sobre la gammagrafía ósea para detectar metástasis óseas, aunque hasta el momento no se ha utilizado de forma rutinaria por escasa disponibilidad de la técnica. Hoy en día es posible realizar un estudio de cuerpo entero (whole body) en RM con menos de 30 minutos empleando secuencias rápidas. Precisamente, nuestro grupo de trabajo es pionero en el estado español en la aplicación de esta técnica con resultados publicados superiores a la gammagrafía ósea (29). Recientemente hemos desarrollado la técnica aplicando una secuencia funcional como es la difusión en RM para realizar el estudio de cuerpo entero. La nueva técnica proporciona imágenes de cuerpo entero con contraste similar al PET (Figura 14). Los resultados iniciales indican una fiabilidad superior de la RM respecto a la gammagrafía ósea (30). Es posible que en el futuro se realice la RM de cuerpo entero como técnica de primera elección en sustitución de la gammagrafía ósea, cuando pueda disponerse de mayores recursos y mayor disponibilidad de la resonancia magnética.

\section{CONCLUSIÓN}

El estadiaje clínico preoperatorio del cáncer de próstata es de escasa fiabilidad. Hoy en día la técnica de imagen de elección para realizar un estadiaje loco-regional del cáncer de próstata es la resonancia magnética en pacientes candidatos a cirugía. La RM ofrece la ventaja de poder combinar la información anatómica y metabólica de la glándula conjuntamente con información ganglionar y del marco óseo en una sola exploración. Los estudios de RM utilizando contraste endovenoso o con partículas de hierro po- 
drían mejorar la capacidad de extensión tumoral y la fiabilidad de la técnica. Así mismo, nuevas técnicas de RM para evaluar el cuerpo entero pueden sustituir la gammagrafía ósea para detectar metástasis esqueléticas. La técnica de RM sigue evolucionando y la posibilidad en el futuro de poder fusionar imágenes de ecografía, RM y PET puede proporcionar un avance en el manejo del cáncer de próstata y establecer nuevas indicaciones de la RM. De todas formas, son precisos más estudios de investigación para poder establecer el papel de la resonancia magnética en el futuro.

\section{BIBLIOGRAFÍA Y LECTURAS RECOMENDADAS (*lectura de interés $y^{* *}$ lectura fundamental)}

1. MINISTERIO DE SANIDAD Y CONSUMO.: "La situación del cáncer en España". Centro de Publicaciones, Madrid, 2005.

2. PARTIN, A.W.; KATTAN, M.W.; SUBONG, E.N. y cols.: "Combination of prostate-specific antigen, clinical stage, and Gleason score to predict pathological stage of localized prostate cancer. A multiinstitutional update". JAMA, 277: 1445, 1997.

*3. KURHANEWICZ, J.; VIGNERON, D.B.; MALES, R.G. y cols.: "The prostate: MR imaging and spectroscopy. Present and future". Radiol. Clin. North Am., 38: 115, 2000.

4. HUCH BONI, R.A.; BONER, J.A.; DEBATIN, J.F. y cols.: "Optimization of prostate carcinoma staging: comparison of imaging and clinical methods". Clin. Radiol., 50: 593, 1995.

5. SCHIEBLER, M.L.; TOMASZEWSKI, J.E.; BEZZI, M. y cols.: "Prostatic carcinoma and benign prostatic hyperplasia: correlation of high-resolution MR and histopathologic findings". Radiology, 172: 131, 1989.

**6. HUSBAND, J.E.; SOHAIB, S.A.: "Prostate Cancer". Husband J.E, Reznek R.H., editors. Imaging in Oncology. 2nd ed. London: Taylor \& Francis, 375, 2004.

7. COMET-BATLLE, J.; VILANOVA-BUSQUETS, J.C.; SALADIE-ROIG, J.M. y cols.: "The value of endorectal MRI in the early diagnosis of prostate cancer". Eur. Urol., 44: 201, 2003.

8. TSUDA, K.; YU, K.K.; COAKLEY, F.V. y cols.: "Detection of extracapsular extension of prostate cancer: role of fat suppression endorectal MRI". J. Comput. Assist. Tomogr., 23: 74, 1999.

**9. VILANOVA, J.C.; BARCELO, J.: "Prostate cancer detection: MR spectroscopic imaging". Abdom. Imaging.,Dec. 5 (E. pub. ahead of print) 2005.
10. VAN DORSTEN, F.A.; VAN DER , G.M.; ENGELBRECHT, M.R. y cols.: "Combined quantitative dynamic contrast-enhanced MR imaging and (1)H MR spectroscopic imaging of human prostate cancer". J. Magn. Reson. Imaging., 20: 279, 2004.

11. VILANOVA, J.C.; COMET, J.; CAPDEVILA, A. y cols.: "The value of endorectal MR imaging to predict positive biopsies in clinically intermediate-risk prostate cancer patients". Eur. Radiol., 11: 229, 2001.

12. COMET, B.J.; VILANOVA, J.C.; MAROTO, G.A. y cols.: "Localization of prostate cancer within the central gland by endorectal MR spectroscopic imaging". Arch. Esp. Urol., 58: 151, 2005.

13. PRESTI, J.C. Jr.; HRICAK, H.; NARAYAN, P.A. y cols.: "Local staging of prostatic carcinoma: comparison of transrectal sonography and endorectal MR imaging". AJR, 166: 103, 1996.

14. WEFER, A.E.; HRICAK, H.; VIGNERON, D.B. y cols.: "Sextant localization of prostate cancer: comparison of sextant biopsy, magnetic resonance imaging and magnetic resonance spectroscopic imaging with step section histology". J. Urol., 164: 400, 2000.

15. CLAUS, F.G.; HRICAK, H.; HATTERY, R.R.: "Pretreatment evaluation of prostate cancer: role of MR imaging and 1H MR spectroscopy". Radiographics, 1: 167, 2004.

16. BEYERSDORFF, D.; DARSOW, U.; STEPHAN, C. y cols.: "MRI of prostate cancer using three different coil systems: image quality, tumor detection, and staging". Rofo, 175: 799, 2003.

17. QAYYUM, A.; COAKLEY, F.V.; LU, Y. y cols.: "Organ-confined prostate cancer: effect of prior transrectal biopsy on endorectal MRI and MR spectroscopic imaging". AJR, 183: 1079, 2004.

18. ENGELBRECHT, M.R.; JAGER, G.J.; LAHEIJ, R.J. y cols.: "Local staging of prostate cancer using magnetic resonance imaging: a meta-analysis”. Eur. Radiol., 12: 2294, 2002.

19. CORNUD, F.; FLAM, T.; CHAUVEINC, L. y cols.: "Extraprostatic spread of clinically localized prostate cancer: factors predictive of $\mathrm{pT} 3$ tumor and of positive endorectal MR imaging examination results". Radiology, 224: 203, 2002.

*20. JAGER, G.J.; SEVERENS, J.L.; THORNBURY, J.R. y cols.: "Prostate cancer staging: should MR imaging be used? A decision analytic approach". Radiology, 215: 445, 2000.

21. POULAKIS, V.; WITZSCH, U.; DE, V.R. y cols.: "Preoperative neural network using combined magnetic resonance imaging variables, prostate specific antigen and Gleason score to predict prostate cancer stage". J. Urol., 172: 1306, 2004. 
22. WANG, L.; MULLERAD, M.; CHEN, H.N. y cols.: "Prostate cancer: incremental value of endorectal MR imaging findings for prediction of extracapsular extension”. Radiology, 232: 133, 2004.

23. WANG, L.; HRICAK, H.; KATTAN, M.W. y cols.: "Combined endorectal and phased-array MRI in the prediction of pelvic lymph node metastasis in prostate cancer". AJR, 186: 743, 2006.

24. YU, K.K.; SCHEIDLER, J.; HRICAK, H. y cols.: "Prostate cancer: prediction of extracapsular extension with endorectal MR imaging and threedimensional proton MR spectroscopic imaging". Radiology, 213: 481, 1999.

*25. WANG, L.; HRICAK, H.; KATTAN, M.W. y cols.: "Prediction of organ-confined prostate cancer: incremental value of MR imaging and MR spectroscopic imaging to staging nomograms". Radiology, 238: 597, 2006.

26. ENGELBRECHT, M.R.; HUISMAN, H.J.; LAHEIJ, R.J. y cols.: "Discrimination of prostate cancer from normal peripheral zone and central gland tissue by using dynamic contrast-enhanced MR imaging”. Radiology, 229: 248, 2003.

27. OGURA, K.; MAEKAWA, S.; OKUBO, K. y cols.: "Dynamic endorectal magnetic resonance imaging for local staging and detection of neurovascular bundle involvement of prostate cancer: correlation with histopathologic results". Urology, 57: 721, 2001.

28. BELLIN, M.F.; LEBLEU, L.; MERIC, J.B.: "Evaluation of retroperitoneal and pelvic lymph node metastases with MRI and MR lymphangiography”. Abdom. Imaging., 28: 155, 2003.

29. BARCELO, J.; VILANOVA, J.C.; VILLALON, M. y cols.: "Técnica de estudio del cuerpo entero por RM para cribado de metástasis óseas en pacientes con neoplasias malignas". Radiología, 46: 359, 2006.

30. VILANOVA, J.C.; BARCELO, J.: "Whole Body Diffusion-Weighted Image: a novel technique to evaluate patients with bone metastases". European Congress of Skeletal Radiology, Bruges (Belgica). 2006. 